\title{
DoReMi workshop on multidisciplinary approaches to evaluating cancer risks associated with low-dose internal contamination
}

\author{
D. LAURIER ${ }^{1}$, I. GUSEVA CANU ${ }^{1}$, S. BAATOUT ${ }^{2}$, J.-M. BERTHO ${ }^{1}$, \\ E. BLANCHARDON ${ }^{1}$, S. BOUFFLER ${ }^{3}$, E. CARDIS ${ }^{4}$, M. GOMOLKA ${ }^{5}$, J. HALL ${ }^{6}$, \\ A. KESMINIENE ${ }^{7}$, M. KREUZER ${ }^{5}$, E. RAGE $^{1}$
}

(Manuscript received 29 November 2011, accepted 13 December 2011)

ABSTRACT A workshop dedicated to cancer risks associated with low-dose internal contamination was organised in March 2011, in Paris, in the framework of the DoReMi (Low Dose Research towards Multidisciplinary Integration) European Network of Excellence. The aim was to identify the best epidemiological studies that provide an opportunity to develop a multidisciplinary approach to improve the evaluation of the cancer risk associated with internal contamination. This workshop provided an opportunity for in-depth discussions between researchers working in different fields including (but not limited to) epidemiology, dosimetry, biology and toxicology. Discussions confirmed the importance of research on the health effects of internal contamination. Several existing epidemiological studies provide a real possibility to improve the quantification of cancer risk associated with internal emitters. Areas for future multidisciplinary collaborations were identified, that should allow feasibility studies to be carried out in the near future. The goal of this paper is to present an overview of the presentations and discussions that took place during this workshop.

Keywords: Cancer / contamination / internal / epidemiology / dosimetry / radiobiology / toxicity / radiological

RÉSUMÉ Approches multidisciplinaires pour l'évaluation du risque de cancer associé aux contaminations internes aux faibles doses : synthèse du séminaire scientifique organisé dans le cadre du projet européen DoReMi.

Un séminaire scientifique dédié à l'étude du risque de cancer associé aux contaminations internes à faibles doses a été organisé à Paris en mars 2011, dans le cadre du réseau d'excellence européen DoReMi (Low Dose Research towards Multidisciplinary Integration). L'objectif était d'identifier les meilleures études épidémiologiques pouvant permettre le développement de collaborations multidisciplinaires afin

\footnotetext{
Institut de radioprotection et de sûreté nucléaire, IRSN, Fontenay-aux-Roses Cedex, France.

Belgian Nuclear Research Centre, SCK-CEN, Mol, Belgium.

Health Protection Agency, Centre for Radiation Chemical and Environmental Hazards, HPA-CRCE, Chilton, UK.

Centre for Research in Environmental Epidemiology, CREAL, Barcelona, Spain

Federal Office for Radiation Protection, Department of Radiation Protection and Health, BfS, Neuherberg, Germany.

Inserm U612, Institut Curie, Orsay, France.

International Agency for Research on Cancer, Section of Environment and Radiation, IARC, Lyon, France.
} 


\begin{abstract}
d'améliorer la quantification des risques associés à une contamination interne. Ce séminaire a été l'occasion de discussions approfondies entre chercheurs de différentes disciplines, telles que l'épidémiologie, la dosimétrie, la biologie et la toxicologie. Ces discussions ont confirmé l'importance des recherches sur les effets sanitaires des contaminations internes. Plusieurs études épidémiologiques existantes présentent une réelle capacité à améliorer les connaissances dans ce domaine. Des pistes de collaborations multidisciplinaires ont été dégagées, qui pourraient déboucher à court terme sur des études de faisabilité. Le but de cet article est de synthétiser les présentations et les discussions qui se sont tenues lors du séminaire.
\end{abstract}

\title{
Context
}

\section{Current issues for research in radiation protection}

Ionising radiation is used for many beneficial purposes in medicine and industry. However, it is recognised that there are health risks associated with exposure to ionising radiation. There is clear evidence that radiation increases the risk of cancer in irradiated populations such as the Japanese atomic bombing survivors when doses are in excess of around $100 \mathrm{mSv}$. Direct evidence of risk below these levels is ambiguous and extrapolation from risk information available from the higher dose studies is used to estimate low dose risk. However, the exposures of concern for human populations are in the vast majority of cases in this low-dose region $(<100 \mathrm{mSv})$ and generally at low dose rates $\left(<1 \mathrm{mSv} \mathrm{min}^{-1}\right)$. It is therefore the health risks in this low dose/dose-rate region that radiation protection standards and policy address. The risk estimates for cancer used in radiation protection derive mainly from epidemiological studies of the Japanese A-bomb survivors (UNSCEAR, 2008). This population was exposed at a high dose rate to external gamma and neutron irradiation, with a wide range of individual doses (from 0 to 4 $\mathrm{Sv}$, with a mean at about $200 \mathrm{mSv}$ ). Many situations of radiation exposure, however, concern internal contamination with radionuclides; not least is the exposure of the public to background radiation, the major source of which is inhaled radon gas. Internal contamination is also encountered in medical diagnostics and in occupational settings. A wide range of radionuclides are potential sources of human exposure. In 2009 the EURATOM-sponsored High Level and Expert Group (HLEG) published a brief report (HLEG, 2009) that considered the key issues in radiation protection policy that required attention and started to develop a research agenda to address these issues.

The main scientific questions that the HLEG identified concern:

1. the shape of the dose-response relationship and tissue sensitivity for cancer,

2. individual variability in cancer risk and genetic susceptibility to cancer,

3. radiation quality (type),

4. internal exposure risk,

5. risks of non-cancer diseases and hereditary effects. 
As such it was recognised that the risks associated with exposure to internal contamination was a key issue requiring further scientific investigation. The five HLEG scientific questions have been taken up as a focus for European research in the short and long term within, respectively, the DoReMi Network (see below) and the Multidisciplinary European Low Dose Initiative (MELODI, www.melodionline.eu/).

\section{The DoReMi European Network}

The DoReMi (Low Dose Research towards Multidisciplinary Integration) European Network of Excellence was launched in January 2010 in the context of the European Commission EURATOM $7^{\text {th }}$ Framework Programme (EC FP7) (http://www.doremi-noe.net/). Its aim is to promote the sustainable integration of low dose risk research in Europe in order to help the effective resolution of current key policy questions. Within this Network, Work Package 5 (WP5) is dedicated to the improved characterisation of the shape of the dose-response for cancer, and includes several tasks that address mechanisms of low dose radiation carcinogenesis and the modelling of risk. Within WP5, Task 5.5 specifically focuses on the risks associated with internal contamination.

\section{Internal contamination and cancer risk}

Exposures to ionising radiation can include both external irradiation and incorporation of radionuclides. The latter usually results in lower doses and lower dose rates than the former. However, it is a major route of public exposure because of radon inhalation, ingestion of naturally occurring radionuclides and possible controlled or accidental discharge from the nuclear industry, and subsequent contamination through drinking water and food. In order to analyse the cancer risks associated with internal exposure, numerous questions linked to the radiological and chemical characteristics of the radionuclides as well as to the need for reconstruction of doses from available monitoring data have to be addressed. The dose reconstruction can lead to large dose uncertainties and greater concerns about the accuracy of risk estimates from incorporated radionuclides compared with those for external radiation. Nonetheless, there are many situations of practical importance where internal contamination is a major source of exposure and these estimates are necessary to be able to assess risk and implement appropriate public health policies. These include the nuclear industry, the underground mining industry, and environmental exposure of populations living on contaminated land.

Internal dosimetry is based on the measurement of activity in the environment and individuals. Biokinetic and dosimetric models represent the behaviour of 
radionuclides in the body and the consequent deposition of energy in the radiosensitive target tissues. Measurement results are translated into a committed effective dose (i.e. cumulated dose that will be delivered over the life span following incorporation) through the application of these biokinetic and dosimetric models. However, significant uncertainties are involved at each step of this process due to counting statistics, variable measurement efficiency, environmental and biological fluctuations, incomplete biokinetic, physical and anatomic datasets, over-simplified models, and heterogeneous distribution of radionuclides, of energy deposition and of target cells in tissues. These uncertainties can lead to discrepancies in dose assessments but can be quantified by mathematical methods such as Bayesian inference (Hofer, 2008; Puncher and Birchall, 2008).

Some epidemiological and experimental results have already demonstrated an increased risk of cancer, mainly at high levels of exposure to several radionuclides. Studies of radium painters showed an excess of bone cancers, and use of thorotrast among patients was linked to an excess of liver cancers (UNSCEAR, 2008). Follow-up of workers employed at the Mayak atomic weapon production plant in Russia identified an excess risk of lung cancer and liver cancer from plutonium inhalation (Sokolnikov et al., 2008). An excess of thyroid cancers has been observed after intake of radioactive iodine among those who were children living in the contaminated territories around Chernobyl (Zablotska et al., 2010; Cardis and Hatch, 2011; UNSCEAR, 2011). A linear exposure-risk relationship has been demonstrated from miner studies and also from large studies in the general population between radon inhalation and lung cancer risk, even at low levels of exposure (BEIR-VI, 1999; Darby et al., 2006; Tomasek et al., 2008). Nevertheless, except for these few exceptions, studies have not yet been able to quantify the dose-risk relationship between internal contamination and cancer risk at low doses.

One of the important research topics in the field of radiation biology is the understanding of the mechanisms related to low doses and the deciphering of the mechanistic link existing between the initial damage and death. This encompasses the search for good early biomarkers of intrinsic radiation susceptibility in order to provide reliable radiation risk factors that would then maybe avoid the need for long-term studies. A thorough review on available biomarker candidates coordinated by E. Cardis and E. Pernot (CREAL, Spain) is ongoing in the framework of DoReMi. Classically, 4 classes of biomarkers have been distinguished: 1) exposure biomarkers (available after exposure and suitable for estimating the dose received), 2) biomarkers of susceptibility (available before, during or after exposure and can predict an increased risk in radiation effects), 3) biomarkers of late effects (that can be used to assess the effects that are present in 
the late stages of a disease caused by radiation) and 4) biomarkers of persistent effects (that allow the assessment of radiation effects that are present a long period of time after exposure). Presently, there is no single biomarker which would be specific to low doses of radiation as well as sensitive in cases of low chronic exposure. There are several reasons that underlie this, including the fact that extrapolation from high dose data to low doses is not straightforward (for example, high doses of radiation are known to induce inflammatory processes whilst low doses can trigger anti-inflammatory response) and that the extrapolation between animal and human studies remains difficult. However, some useful biomarkers already exist for exposure (such as chromosomal aberrations) and for early biological effects (such as DNA single-strand breaks monitored by the comet assay, and DNA double-strand breaks assessed by the formation of gamma-H2AX and micronuclei). Certain other classes of biomarkers are much more in the developmental stages, such as, for instance, biomarkers of individual sensitivity such as genetic variants in candidate genes that will influence cellular responses to radiation exposures. New technologies such as high-throughput gene sequencing, epigenomics, transcriptomics and other omics are promising approaches that are being used to test and identify radiation biomarkers with high specificity and sensitivity. In addition, non-genetic markers such as vitamins, antioxidants, lipids and immunoglobulins may be useful biomarkers of past exposure but their specificity with respect to radiation exposure is expected to be low. A major effort is being made at the European level so that these potential biomarkers can be validated. Other biomarkers may also be relevant for increased cardiovascular risk (dependent or not on radiation), such as 1) inflammatory markers such as white blood cell count, C reactive protein, Interleukin 1 RA, Interleukin 6, CD40/CD40L, TNF-alpha and Troponin (heart tissue necrosis marker); 2) genetic markers such as 9p21.3, 6q26-27, 21q22, 2q33, 3q22.3, 12q24, 6p24, 1p13.3, $10 \mathrm{q} 11,1 \mathrm{q} 41$ and 12q24.3, and 3) biochemical markers such as serum cholesterol (LDL-cholesterol and HDL-cholesterol) and triglycerides (Little et al., 2009; AGIR, 2010; Bonatera et al., 2010; Erdmann et al., 2010).

A number of specific issues related to internal dosimetry and radiobiology associated with epidemiological studies can hamper study outcomes. These include difficulties associated with investigating historical working conditions in routine situations or in the case of an event, loss of knowledge of historical measurement techniques and specifications, loss of measurement results, and lack of ambient/individual data. In addition, most exposures are below the limit of detection of the bioassays (individual measurement of radionuclides in the body or in excreta) used to date. These limitations compound the large and imprecise uncertainties in doses. Problems associated with the preservation of biological samples, the lack of appropriate biomarkers, and the ethical and social issues of 
obtaining biological samples also limit the development of molecular epidemiological studies related to internal exposure. Finally, it must also be recognised that epidemiological studies have limitations, such as insufficient statistical power, biases, uncontrolled confounding factors, or insufficient length of follow-up.

\section{Organisation and objectives of the workshop}

The DoReMi Task 5.5 workshop, entitled "Multidisciplinary approaches to evaluating cancer risks associated with low-dose internal contamination" was held on the 24-25 March 2011, at the "Cité internationale universitaire" in Paris (programme available at www.doremi-noe.net/). This workshop was organised by the French Institute for Radiological Protection and Nuclear Safety (IRSN), with the support of the UK Health Protection Agency (HPA) and the French "Commissariat à l'énergie atomique" (CEA).

\section{Objectives of the workshop}

The general aim of the workshop was to identify the best epidemiological studies that provide an opportunity to develop a multidisciplinary approach to improve the evaluation of the cancer risk associated with internal contamination. The workshop aimed to provide an opportunity for in-depth discussions between researchers working in different fields including (but not limited to) epidemiology, dosimetry, biology, toxicology and modelling.

Specific objectives were to evaluate the quality of the available data and the possibilities of collecting additional data, to assess possible linked experimental studies and their requirements for data and/or biological samples and the possibilities of developing collaborative research programmes in Europe.

\section{Organisation of the workshop}

Participation in the workshop was upon invitation, with the number of participants limited to 50. Participants were selected from among researchers in the fields of epidemiology, dosimetry, biology, toxicology and modelling, with a specific interest in internal contamination, within and outside the DoReMi network.

The objective of the workshop was to consider specific exposure situations selected on the basis of a critical review of the existing cohorts. A summary table of main epidemiological studies in the field of internal contamination was produced before the workshop and distributed to all participants, on the basis of 
the review coordinated by Elisabeth Cardis (CREAL, Spain) as a part of DoReMi WP4.

The workshop included both invited presentations and discussions in parallel working groups. Talks were invited in four main sections:

- context and objectives;

- main issues related to cancer risk and internal contamination;

- experiences of multidisciplinary collaboration in this field;

- methodologies and structures of interest.

In order to allow discussions in smaller groups, three parallel working groups were predefined to consider specific topics:

- workers employed in the nuclear industry;

- Chernobyl liquidators and Techa river residents;

- uranium miners.

A list of questions, focused on criteria to identify the best opportunities for multidisciplinary investigations, was distributed beforehand to each participant. This list (available at www.doremi-noe.net/) was used in each of the three working groups to structure the discussions and facilitate the combination of the results of the parallel discussions.

\section{Summary of the presentations}

The details of all invited presentations described below are available on the DoReMi website (www.doremi-noe.net/).

\section{Context and objectives}

As the coordinator of the DoReMi European Network of Excellence, Sisko Salomaa (STUK, Finland) presented the European context of research in the field of radiation protection. She reviewed the main objectives of the DoReMi project, its organisation and its relationship with the MELODI initiative. She underlined the importance of research on the effects of internal emitters, which constitutes one of the cross-cutting issues in the DoReMi project.

Simon Bouffler (HPA, UK), the leader of the DoReMi WP5, summarised the objectives of this WP dedicated to the shape of the dose-response relationship for cancer. The aim of this WP is to improve knowledge of low dose cancer risk in humans. It consists of five Tasks, focused respectively on phase shifts in the doserisk relationship, non-targeted phenomena, pre-neoplastic/clonal development, mathematical modelling of cancer risk, and risk from internal exposure. 
The workshop used as an input for discussion a review of the main epidemiological studies in the field of internal contamination. This review was derived from a larger one, performed in the framework of WP4, that considered all cohorts of individuals exposed to ionising radiation. Elisabeth Cardis (CREAL, Spain) presented the methods used and the results of this review. Data from about 55 European cohorts that are being used to assess risks (cancer and non-cancer endpoints) following radiation exposure were collected from standardised questionnaires. Among these studies, 45\% concerned occupational exposures, $40 \%$ involved exposures in the medical field and $15 \%$ dealt with environmental exposures (Cardis and Pernot, 2011).

The aim of WP5 Task 5.5 was detailed by Dominique Laurier (IRSN, France). Issues related to the epidemiological, dosimetric, toxicological and biological aspects were summarised.

\section{Main issues related to cancer risk and internal contamination}

Dominique Laurier presented the review of epidemiological studies of long-term cancer risk associated with internal contamination. The advantages and limits to, and the pre-requisites for such epidemiological studies were detailed. A total of 27 studies dealing with internal contamination in Europe was reviewed. The majority of these studies (60\%) were conducted on cohorts exposed occupationally (miners, workers in the uranium fuel cycle). The most informative studies can be defined as those with greater statistical power, good quality and long duration of followup, and individual exposure data. With respect to their ability to quantify a doserisk relationship for internal contamination, the occupational studies, in which precise individual exposure measurements are collected, appear of major interest. However, other studies, such as cohorts of medically exposed patients, may also be valuable and informative. Dominique Laurier also presented the point of view of the epidemiologist with respect to what can be gained from the use of biomarkers in epidemiological studies, namely an increase in the precision and accuracy of the estimations of relative risks through a better classification of the individuals according to the disease or exposure.

In the absence of John Harrison (HPA, UK), Simon Bouffler presented a general review of dosimetric aspects linked to internal contamination and their integration into the current radiation protection system (Harrison, 2009). He summarised the existing biokinetic and dosimetric models, and the different steps in the estimation of a dose. He highlighted the research priorities, in epidemiology, in the experimental field and in dosimetric models. The SOLO European project, dedicated to the analysis of risk in the Russian Mayak workers and Techa river residents, was also introduced. 
The presentation of François Paquet (IRSN, France) gave an overview of toxicological and dosimetric aspects of Auger and beta emitters. The talk was illustrated by the examples of tritium and iodine toxicity and their relative biological efficiency. The issues raised by Auger and other short-distance emitters were discussed, regarding the cellular dosimetry and its integration into the current radiation protection system (Paquet et al., 2011).

Nick Priest's (AECL, Canada) presentation focused on the effects of boneseeking alpha emitters, especially radium and plutonium (Priest and Hoel, 2010), considering both epidemiological and experimental results. Issues in the modelling of the relationship between osteosarcoma risk and skeletal dose were discussed. He suggested that a threshold effect at low doses may exist, if the lag time between exposure and effect becomes greater than the life duration of the exposed individuals.

\section{Experiences of multidisciplinary collaboration in this field}

Harmen Bijwaard (RIVM, The Netherlands) presented examples of biologicallybased modelling of cancer risk associated with internal contamination. Examples of the parallel application of carcinogenesis models to both animal and human data were presented for both plutonium exposure and bone cancer, and radon exposure and lung cancer (Bijwaard and Dekkers, 2007; van Dillen et al., 2011). This approach appears to be a comprehensive way to combine data from biology and epidemiology in order to produce new information about the shape of the doseresponse for cancer.

The Techa river resident population (Urals, Russia) has been exposed to radioactive releases from the Mayak weapon production plant since the 50s. Longterm collection of both biological and epidemiological data from this population has been performed. Alexander Akleyev (URCRM, Russia) presented recent results on haematopoietic effects of chronic radiation exposure obtained from the Techa river cohort. These data have allowed the analysis of the dynamics of blood and red bone marrow cell counts as a function of dose, and to evaluate the pertinence of these biological modifications with respect to the risk of leukaemia occurrence (Akleyev et al., 2010a, 2010b; Akushevich et al., 2011). These results constitute a very good illustration of the integration between epidemiology and biology, and highlight the usefulness of biological information in the estimation of the radiation-induced risk of cancer in a population.

Maria Gomolka (BfS, Germany) presented the ongoing research into bioindicators among the German Wismut cohort of uranium miners. Four different projects have been launched, with the aim of studying biomarkers of chronic 
radiation exposure in the past, to identify biomarkers in correlation with radiationinduced diseases, to detect genetic factors contributing to individual radiation susceptibility and to investigate biological effects induced by combined exposure of ionising radiation and other toxic compounds, such as arsenic. Collected samples allow investigation of genetic and epigenetic radiation effects as well as the investigation of proteomic indicators. In addition, cell samples were collected and cryo-conserved to also perform bioassays. Preliminary results demonstrated the feasibility of blood sample collection in the framework of the Wismut miner cohort and the high quality of the biological material. Transportation conditions and sampling protocols were evaluated (Weber et al., 2010) and optimised for feasibility in epidemiological cohorts.

The presentation by Margot Tirmarche (IRSN, France) described collaborative research projects on radon risk implemented in EC FP5 and FP6. These projects relied on close collaboration between epidemiologists, biologists and dosimetrists. Under FP5, a parallel analysis of lung cancer risk from low-level radon exposure from animal and human data was undertaken (Tirmarche et al., 2003). In the FP6 Alpha-Risk project (www.alpha-risk.org), the collaboration between dosimetrists and epidemiologists allowed the estimation of cancer risk among uranium miners based on organ dose calculations (Tirmarche et al., 2010).

\section{Methodologies and structures of interest}

Janet Hall (Institut Curie, France) introduced the background and methodology of molecular epidemiology. Several different approaches (genetic, genomic, transcriptomic, proteomic, epigenetic, etc.) have developed rapidly in recent years and different aspects (technical, scientific and ethical) related to biomarker research were reviewed. Molecular epidemiology appears today to be a very promising approach, bringing new biological information on an individual basis to complement epidemiological studies, in order to provide new information on the effects of radiation at low doses. The issue was raised of the pertinence of blood samples for internal contamination, and the need for biomarkers of exposure specific to different target organs.

The STORE platform was presented by Bernd Grosche (BfS, Germany). This project, launched in 2009 through the EC FP7 programme, aims to develop a data/sample warehouse to store and share material, data and the results from research performed in the field of ionising radiation. Such a platform would allow access to data and biological archives that could be of use for the better understanding of the effects of internal contamination at low doses. 
Isabelle Testard (CEA, France) presented the review of existing infrastructures of interest for research projects investigating the impact of internal contamination in Europe. This review drew on information gathered in a larger survey performed within the framework of the DoReMi WP4 under the coordination of Laure Sabatier (CEA, France). It appeared that external irradiation installations are preponderant, but only four facilities in Europe allow the experimental contamination of animals: two in France at IRSN and CEA, one in the UK at HPA and one in Germany at HMGU (the latter will be closing in the next 5 years). Large facilities exist outside Europe like, for example, in Canada at AECL.

\section{Parallel working groups}

The objectives of the three parallel working groups encompassed the examination of different issues regarding epidemiological, dosimetric and biological aspects of currently available data in Europe, to address their potential to give some insight into the shape of the dose-response relationship, to define the priorities in filling the existing data or knowledge gaps, and to examine how collaboration in the context of the DoReMi and/or MELODI networks would be helpful to steer new investigations.

\section{Working group on nuclear workers}

The first working group was dedicated to nuclear worker studies. It was chaired by Irina Guseva-Canu (IRSN, France) and Sarah Baatout (SCK-CEN, Belgium).

\section{Epidemiological issues}

The most common radionuclides involved in internal contamination events of nuclear workers are actinides and tritium. Since several European and US scientific programmes were and are specifically devoted to plutonium (SOLOproject, SOUL-project), this radionuclide was considered beyond the scope of the present workshop. The information on the statistical relationship between occupational exposure to uranium and cancer incidence, or cancer and non-cancer mortality is scant (Guseva Canu et al., 2008a). The UNSCEAR 2006 report clearly states the current position based on scientific evidence: "there is little or no epidemiological evidence for an association between uranium and any cancer" (UNSCEAR, 2008). Nevertheless, most studies conducted up to now were limited in their capacity to demonstrate an associated risk, mainly due to their reduced statistical power or to the poor quality of their dosimetric data (Guseva Canu et al., 2008a). However, new studies have been recently launched in the US based on updated cohorts with comprehensive exposure assessment and internal dose 
estimation and have already provided informative results with respect to the lymphatic and haematopoietic tissue malignancies (Yiin et al., 2009; Chan et al., 2010; Boice et al., 2011). In Europe, a similar study was conducted in France, in the framework of a pilot investigation; though internal doses have been only partially assessed. This study suggested an exposure-response relationship for lung cancer (Guseva Canu et al., 2010a) and lymphatic and haematopoietic tissue malignancies (Guseva Canu et al., 2011). Another study, using a nested casecontrol study design, has been launched in the framework of the EC FP6 AlphaRisk research project, and is still in progress (Tirmarche et al., 2010). The objective of this study was to estimate the risk of lung cancer and leukaemia among French, Belgian and UK nuclear workers who were employed in occupational settings where they were potentially exposed to uranium or plutonium.

Today, the most complete epidemiological information is available from nuclear workers in the UK, France and Belgium. It should be noted that although the data related to the UK nuclear workers are managed by different national institutions depending on the plants and activity, they should be considered all together, like the UK cohort (Carpenter et al., 1998; Atkinson et al., 2004; McGeoghegan et al., 2008). The UK workers have been monitored for certain radionuclides, including tritium, plutonium, and sometimes uranium. Some bioassay data are readily available, while no data exists on chemical co-exposure and lifestyle factors, such as smoking.

The use of the French nuclear worker cohorts for molecular epidemiological studies has the advantage that an individual biomonitoring for uranium exposure is available (Guseva Canu et al., 2010b). These biomonitoring data and medical surveillance data are partially computerised. Moreover, for some groups of workers quantitative or semi-quantitative information on the physical and chemical form of uranium as well as co-exposure to chemicals was assessed (Guseva Canu et al., 2009). However, no biological data are available except the results of routine biochemical analyses of blood. Belgian workers also benefited from an individual biomonitoring for uranium exposure, and these data are readily available for almost all workers. In total, the workers of the FBFC (Franco-Belge de Fabrication du Combustible) plants (Pierrelatte and Roman in France and Dessels in Belgium) represent about 4,000 individuals. The population of workers involved in the nuclear fuel cycle could provide a very good opportunity to evaluate the possibility of constructing a combined cohort (compatibility of databases), with a precise reconstruction of past exposures to insoluble uranium oxides and other exposures (availability of data, job exposure matrix construction), including the feasibility of biological sampling and biomarker testing (legal and 
logistic procedures), and the collection of additional information (hypertension, serum cholesterol levels, etc.) from the occupational medical files.

Taking into account the limits/advantages of the different cohorts in terms of size, duration of follow-up, data quality and completeness, and availability of additional information, no single cohort could provide a satisfactory dose-response relationship adjusted for smoking and other occupational carcinogens. However, the pooling of the available data using common study protocols should provide sufficient statistical power. The discrepancies in biomonitoring and medical surveillance between countries are an issue and should be addressed in the protocol, as well as the authorisations of national ethical committees and local trade unions.

\section{Exposure and dosimetric issues}

Among the exposure issues, the workshop participants underlined the importance of the characterisation of the physical and chemical form of the compounds (aerosol size, solubility, etc.), which are closely related to the atmospheric measurement data from individual and/or workstation exposure monitoring. In some studies, plant-specific job exposure matrices (JEM) would partially compensate for a lack of such information (Guseva Canu et al., 2010b). JEM would also be helpful to assess the co-exposure to other, non-radiological carcinogenic or toxic pollutants (Guseva Canu et al., 2008b, 2009).

For radiological pollutants, the activity should be estimated in $\mathrm{Bq}$, to keep consistency over time, in spite of model and software evolution. Organ-specific absorbed doses, in Gy, must be assessed and calculated for the dose-response analyses. Target organs (i.e. those at increasing risk of disease) should be considered in priority: lung, red bone marrow and brain, and then kidney and lymph nodes. The contribution of internal contamination to total dose may not be negligible and depends on the type and isotopic composition of radioactive compounds. According to available Belgian and French estimations, doses to the lung from incorporated uranium may be on average about 5-10 mGy (Guseva Canu et al., 2010b). The organ-specific doses should be computed using a standardised procedure. Some related dosimetric issues have already been considered within the EC FP6 Alpha-risk project, which led to a common EnglishFrench dosimetric protocol (Tirmarche et al., 2010). The quality of old uranium bioassay data remains challenging. Nevertheless, since the 80s, more sensitive methods have been implemented, such as mass spectrometry, resulting usually in more accurate data. Regarding the dose estimation approach, quantification of uncertainties and available software, the experience of the Sellafield (BNFL) study would be the most informative. Indeed, bioassay data of UK and Belgian workers 
are already computerised, while French data computerising is still in progress. The IMBA professional plus software (HPA, UK) was used to estimate life-long individual doses with associated uncertainties, in the same way in samples of French, English and Belgian workers within the Alpha-Risk project (Thierry-Chef et al., 2010). A better assessment of the physical and chemical form of uranium compounds would refine the dose estimates and reduce uncertainties.

\section{Biological issues}

As already stated, the search for biomarkers is one of the most important research topics in the field of radiation biology today. Presently, there is no single biomarker which would be specific to low doses of radiation as well as sensitive in cases of low chronic exposure. However, several existing biomarkers of exposure or of early biological effects could prove pertinent for internal contamination and cancer or non-cancer diseases.

The review of existing epidemiological studies showed that biological data from exposed workers are very scarce. Outside the cohort of Mayak workers (which was considered as out of the scope of the workshop as it is already involved in the SOLO EC project), only a few blood samples were collected in the UK on a small proportion of the BNFL cohort of workers.

It was mentioned during the discussion that for the French-Belgian workers, data on hypertension, cholesterol, triglycerides and other biochemical analyses of blood, plasma and urine are already available. These data could prove of great interest to analyse cardiovascular risk associated with radiation, also taking into account additional well-known risk factors.

In planning for a molecular epidemiological study, the ideal situation would be to perform repeated sampling from the same person at different times during the follow-up. This would enable the follow-up of the variation pattern of markers with time in correlation with disease onset. The importance of confounding markers (age, gender, any previous exposure, and others) should not be neglected. Biological sampling should be the least invasive possible. It was noted that a main constraint in this kind of investigation at the present time is the lack of long-term financial support that would provide multi-sampling over a long period of time (since cancer as well as non-cancer diseases have a latency of decades).

\section{Collaboration issues}

All participants agreed that only collaborative multi-country epidemiological studies with reliable internal dose estimates could provide insights relevant to 
radiation protection issues. Several possibilities of developing collaborative epidemiological studies in Europe were identified. For instance, pooling data from the UK, Belgium and France: combining different disciplines, especially dosimetry and epidemiology, to improve exposure estimates, improving the collaboration between industrial hygienists, occupational physicians and epidemiologists to assess the exposure to radionuclides and non-radioactive pollutants (i.e. chemicals, fibres) would all be beneficial. In addition, carrying out nested case-control studies would probably be very relevant to quantify the doseresponse relationship. Certain biological markers that have been validated in other settings could be incorporated into such studies, starting with feasibility studies on a small nested cohort. For instance, the assessment of the use of biomarkers of exposure or bioassays to assess the variation in responses, and thus potential molecular defects due to internal exposure, using lymphocytes from blood samples could be envisaged. Mechanistic modelling studies would also be possible.

Internal contamination among British, French and Belgian nuclear workers mainly occurs with uranium. In the UK, exposure of workers to tritium is also of concern, but epidemiological data on uranium are limited and almost absent for tritium. Concerning uranium, there is a clear possibility of collaboration and integration of the specific competences found in the different centres: JEM development and exposure characterisation from France; dosimetric software and error measurements and uncertainties from the $\mathrm{UK}$, and ongoing biological studies and available methodology and techniques from Belgium.

\section{Working group on Chernobyl liquidators and the Techa River}

The second working group was mainly dedicated to considering accidental exposures, especially those that occurred among Chernobyl liquidators (clean-up workers) and Techa river residents. It was chaired by Ausrele Kesminiene (IARC, France) and Jean-Marc Bertho (IRSN, France).

\section{Epidemiological issues}

To date, approximately 500,000 Chernobyl liquidators are registered in the national registries of Belarus, Estonia, Latvia, Lithuania and the Russian Federation. However, only those who worked on the industrial site close to the damaged reactor in the first weeks following the accident on 26 April 1986 and/or those who were sent to the $30-\mathrm{km}$ zone for clean-up activities from the contaminated areas of Belarus and Ukraine and consumed contaminated products after returning home from work in April-June 1986 were potentially exposed internally via an intake of radioactive isotopes (iodines and caesium mainly). The 
early liquidators originating from several countries were exposed via airborne radioisotopes, while the intake through ingestion occurred only among the liquidators from the contaminated regions of Belarus and Ukraine. The largest group (approximately 23,000 liquidators) who worked on the industrial site close to the damaged reactor in April-May 1986, when the concentration of radioiodines in the air was very high and therefore intake through inhalation was possible, is from the Russian federation. Another group of liquidators that was contaminated through ingestion is from Ukraine and includes approximately 40,000 liquidators. There are individuals from the Baltic countries and Belarus who were also included in cohorts of liquidators with internal contamination but the numbers from these countries are relatively small. A passive follow-up of these sub-cohorts is possible through the existing Chernobyl and Cancer registries. Until now, only one study (Kesminiene et al., Submitted) has made an attempt to evaluate the risk of thyroid cancer in Chernobyl liquidators from both external exposure and intake of radioactive iodine. Further studies of this population may substantiate data for addressing effects of exposure to radioactive iodines in adulthood.

The Techa River cohorts are composed of 30,000 adults and 24,000 offspring, with a follow-up from the 1950s up to 2005. (Kossenko et al., 2005; Akleev et al., 2007). Both cancer incidence and mortality have already been described, but other issues (cardiovascular diseases, effects of internal contamination on the immune system) could be studied in the future. For instance, a case-control study of cardiovascular diseases nested within the Techa River cohort would be of great interest, although it would require the collection of information about confounding risk factors. These studies are in addition unique in as much as trans-generational studies (up to F2 generation) are possible. The Techa River cohort was exposed to a mixture of radionuclides, including 137-Caesium and 90-Strontium, mainly through ingestion. One caveat should, however, be kept in mind: because of the recent changes in the national regulation of the Russian federation with respect to the protection of personal information, the follow-up of this cohort may become more complicated.

There are several other cohorts that could be informative for studying various aspects of the effects of internal exposure to radioactive iodines:

- The Belarusian-American (Bel-Am) (Zablotska et al., 2011) and the UkrainianAmerican (Ukr-Am) (Ostroumova et al., 2009) cohorts include approximately 25,000 children exposed to radioactive iodines from the Chernobyl fall-out. Individual 131-Iodine thyroid doses as well as the doses to other tissues were estimated for each member of the two cohorts based on individual radioactivity measurements taken within two months after the accident. Until now mainly 
thyroid cancer and other thyroid diseases such as autoimmune thyroiditis (Tronko et al., 2006) and hypothyroidism (Ostroumova et al., 2009) have been studied in these cohorts but the follow-up period is relatively short. This joint Bel-Am - Ukr-Am cohort is a unique source of information not only for the study of thyroid diseases but also for a number of other cancer and non-cancer outcomes for which screening is needed in a well-defined population with individual dose estimates.

- There are two unique cohorts of Chernobyl thyroid cancer patients treated with 131-Iodine for thyroid cancer at very young ages - one in Belarus and another in Ukraine (Tuttle et al.) including altogether about 3,000 patients. The risk of occurrence of secondary cancers (such as breast, lung cancer or lymphoma) but also of non-cancer diseases in this cohort is of interest as this is the largest cohort that was treated with 131-Iodine very early in life, thus allowing the evaluation of the life-long risks of cancer and non-cancer diseases from this radiotherapy. However, most of these patients received a double internal exposure (from the Chernobyl fallout and from the thyroid treatment) which may cause difficulties in assessing which exposure initiated the outcome to be studied.

- The Venus study (France, Italy and Sweden) is a medical cohort of 11,000 patients treated with 131-iodine for thyroid cancer and other thyroid diseases, with on average 14 years of follow-up (Rubino et al., 2003; Garsi et al., 2008). This study could be informative to assess the effects of therapeutic exposure to 131-iodine on tissues and organs other than the thyroid. Depending on tissue availability, the genetic profile of thyroid cancers could be studied in this cohort and compared with the results from recent studies of small numbers of patients with external thyroid irradiation (Ory et al., 2011) or with internal exposure to radioactive iodines following the Chernobyl accident (Port et al., 2007).

The cohort of sarcophagus workers, including about 2,000 workers exposed in recent years (since 2004) was also discussed, but appears to be out of the scope of this discussion as the exposure is mainly external.

\section{Exposure and dosimetric issues}

For most of these cohorts, extensive exposure and/or dosimetry reconstruction has already been done. For instance, the quantity of administrated 131-Iodine is well documented in the medical cohorts, although as mentioned previously, the radiation dose to the target organ (thyroid) is very high. Much effort has gone into the evaluation of the exposure of the Techa River cohort members; additional work to reduce uncertainties in the organ doses reconstructed for this cohort is currently in progress (Tolstykh et al., 2011). Individual doses to the thyroid from the 
radioactive isotopes of iodine as well as the doses to other organs outside the thyroid that were reconstructed based upon individual thyroid activity measurements are also available for the members of the Bel-Am and Ukr-Am cohorts. Dose reconstruction from external radiation and from intake of long-lived radionuclides has been successfully conducted in nested case-control studies of liquidators using Realistic Analytical Dose Reconstruction with Uncertainty Estimation (RADRUE) (Kryuchkov et al., 2009; Drozdovitch et al., 2010). A foreseeable challenge is the reconstruction of doses from internal emitters, as the method used in the case-control study (Kesminiene et al., Submitted) was validated based on a limited number of measurements of radioactive iodines in the air above the reactor and on a limited number of individual thyroid activity measurements performed on the liquidators in the early post-accident days. Doses from 131-Iodine need to be reconstructed based on available information on thyroid activity measurement, aerosol composition and job-exposure matrix for the liquidators involved in the clean-up activities in April-June 1986. Nevertheless, the available dosimetry is of good quality in most of these cohorts discussed above. Moreover, these cohorts were mainly exposed to radionuclides that are not known to present chemical toxicity (such as iodines, caesium, and strontium) which could have a possible confounding effect.

\section{Biological issues}

Thyroid tumour samples are available from the Bel-Am and Ukr-Am cohorts. These samples may allow studies on genetic profiling, individual sensitivity and possibly radiation dose relationship. Samples were also collected from some Chernobyl Ukrainian liquidators. Tissue repositories exist for the medical cohorts, although availability of tissues for the Venus cohort may be limited, since the cohort was established in the 1950s.

About 4,300 samples (blood, tumour tissue, normal tissue, teeth, autopsy samples, etc.) are available in a biological repository from the members of the Techa River cohort. In addition, blood samples from the 3 generations of 100-150 families have also been collected and stored, allowing the setting up of studies of trans-generational effects and possibly reproductive effects in the Techa River resident population.

However, it is not clear what endpoints can be studied using the available biological material. Obviously, since most of the exposures were received several years ago, the study of markers of exposure is unlikely to be informative. Biological samples should be therefore used preferentially for screening issues, for genetic profiling of tumour tissues, for the search for genetic markers of some specific diseases such as leukaemia, or for metabolomic experiments which may 
highlight pathways for some non-cancerous diseases, e.g. cardiovascular diseases. The use of these existing biological repositories needs to be more extensively discussed between epidemiologists and biologists in order to more clearly delineate what is feasible. The quality of samples also needs to be evaluated.

\section{Collaboration issues}

Overall, there is a potential in future use of the Chernobyl and Techa River cohorts for assessing risks associated with internal exposure. Continuation of active follow-up of the Bel-Am and Ukr-Am cohorts should be discussed in collaboration with the American partners and with the EC to avoid duplication and to plan pooling of the resources. These studies could envisage the use of biological material collected during previous screening cycles. Studies of the Chernobyl liquidators require very close collaboration between epidemiologists and dosimetrists; there is also a potential for collaboration with the American partners who are planning to start a case-control study of thyroid cancer among the Ukrainian liquidators. Further studies of leukaemia risk in relation to blood parameters in the Techa River population would be valuable, based on a tight collaboration between biologists, epidemiologists and statisticians. Very close collaboration between various disciplines and partners in summarising all available information on ongoing and planned studies is essential in order to use the available resources in the most efficient way.

\section{Working group on uranium miners}

The third working group was dedicated to uranium miner studies. It was chaired by Michaela Kreuzer (BfS, Germany) and Estelle Rage (IRSN, France).

\section{Epidemiological issues}

Uranium miner cohorts are relevant to study the risks associated with internal contamination due to chronic occupational exposure to inhaled radon gas, radon progeny and uranium ore dust particles. In Europe, most uranium mines are closed now (except in the Czech Republic), but follow-up is still in progress. There are three cohorts of uranium miners, the Czech $(n=10,000)$, the French $(n=5,000)$ and German $(n=60,000)$ cohorts. The cohorts are characterised by a large size, early start dates (between the 1940s and 1950s), a long follow-up period (more than 30 years on average), a small percentage of participants lost to follow-up, and a large number of cancer and non-cancer deaths (Tomasek et al., 2008; Vacquier et al., 2008; Kreuzer et al., 2010). Individual information on radiation exposure (radon progeny, long-lived radionuclides, and external gamma radiation) is 
available for all cohort members and organ doses have already been calculated for several target organs.

With respect to potential confounders, information on smoking habits for subgroups of the three cohorts was collected from nested case-control studies on lung cancer (Leuraud et al., 2007; Schnelzer et al., 2010; Leuraud et al., 2011; Tomasek, 2011). Additional information on potential occupational confounders (e.g. silica dust, arsenic) is available from the German cohort. Nevertheless, limitations exist, such as the lack of information on other potential confounders such as indoor residential radon or medical X-ray exposure with respect to leukaemia or e.g. blood pressure with respect to cardiovascular diseases. Presently, European studies have mainly focused on cancer and non-cancer mortality, but incidence could be relevant to study specific diseases such as leukaemia. Data on cancer incidence could be collected, but only partially within the existing cohorts.

In addition to the European miner studies, there are also several radon-exposed miner studies in Canada (Ontario, Eldorado, Newfoundland), the USA and China, some of which are still active (follow-up of mortality still in progress). An international pooling project of the European with the Canadian cohorts is still in progress and could be based on the experience of the previous European collaborative project of Alpha-Risk. The large size of such a collaborative project could improve the statistical power. This would be particularly useful for investigating the risks associated with low doses for diseases other than lung cancer. It has to be ascertained whether other cohorts (USA, China, etc.) could be integrated.

\section{Exposure and dosimetric issues}

Individual data on radon progeny, uranium ore dust and gamma exposures are available for the French, German and Czech cohorts. Different methods of exposure assessment have been used amongst the cohorts and these have changed over time. Detailed expert rating was performed in the early years (before 195055). Later, exposure reconstruction was based on ambient air measurements (jobexposure matrix) or personal dosimeters (for France), leading to less uncertainties. Considerable efforts have been made in the field of reconstruction of exposures during the last 25 years. New approaches should focus now on measurement error assessment and uncertainties: detailed uncertainty analyses are in progress for the French cohort regarding the assessment of measurement error and the assessment of its impact on the estimated lung cancer risk. Similar analyses should be extended to the other cohorts. 
Organ doses have already been calculated in the European cohorts in the framework of the EC FP6 Alpha-Risk project (Tirmarche et al., 2010). Calculations were performed for five organs: lung, kidney, liver, stomach and red bone marrow. Presently, no dose calculation has been done for the Canadian cohorts. The estimation of the doses was based on different exposure scenarios (job type, physical activity, ventilation, use of diesel machineries, particle size distribution of radon progeny, etc.) and ICRP dosimetric models. Absorbed doses for radon gas, radon progeny and long-lived radionuclides were calculated by ad hoc software (Marsh et al., 2008, 2011). Other target organs could be of interest such as the heart or blood vessels, depending on the disease being considered. Calculated doses allow separate consideration of the contribution of internal and external exposures. The contribution of internal contamination is organdependent, e.g. for the lung, the main contributor is radon progeny, whereas for the organs outside the respiratory tract, the main contributor is external gamma rays. In order to combine alpha and non-alpha radiation, different weighting factors could be considered. Uncertainty analyses on doses have not yet been performed, and this could be an important point for future work on dosimetry.

Beyond radiological effects, some exposures could present toxicological effects. This is not an issue for radon exposure, but exposure to uranium dust with the potential toxicity of uranium in the kidney, for instance, could be considered. The physical-chemical characteristics of aerosols have been used by dosimetrists to calculate organ doses. Regarding confounders, these characteristics could also be relevant to study the effect of dust or silica exposure.

\section{Biological issues}

Pertinent biological indicators are biomarkers of exposure, of susceptibility and of early effects. Particular need was seen for the development of biomarkers of exposure valid for long duration and chronic exposures, since such biomarkers do not exist today. Electron Paramagnetic Resonance Spectroscopy might be a promising method. In addition, as mentioned above, biomarkers of radiation susceptibility are currently being sought through research in many different systems, although at the moment, there is no validated marker to identify radiationsensitive individuals applicable on a population basis to individuals exposed to low doses of radiation. A number of promising biomarkers or biomarker assays exist, such as gamma-H2AX and apoptotic markers, that could be evaluated in pilot studies. Longitudinal cohort studies including repeated sample collection over time are of great interest to detect markers of early effects. However, due to the old age of highly exposed individuals, such biomarker research will be more and more difficult. 
The assessment of leukaemia risk among uranium miners is one of the main issues in current epidemiological studies. As the frequency of specific chromosomal translocations is correlated with leukaemia incidence, one possibility to validate this event as a biomarker for cancer risk would be to quantify translocations in cells of exposed versus non-exposed individuals. It has to be noted though that there will be many issues to address before such a study can be undertaken.

There have been various in vitro and in vivo animal studies on rats and dogs exposed to radon, small particles and smoking to investigate the cancer risks associated with such exposures (Monchaux et al., 1994, 1999; Hofmann et al., 2006). If biological samples are still available and appropriately stored from such studies, they would offer one possibility to develop and validate biomarkers before testing their applicability in human miner cohorts.

For the German uranium miners, biological samples (blood, DNA, plasma, RNA, lymphocytes) have already been collected for 400 healthy miners with high and low radon exposure, and for 82 lung cancer cases (DNA, blood). For 700 lung cancer cases out of the pathology archive, isolation of DNA and partially RNA from paraffin embedded tissue samples is currently being performed. Similar approaches could be envisaged in the Czech and French cohorts, at least for a subsample of miners. Biobanking in France should be launched rapidly for previous miners who are still employed at AREVA, according to a standardised protocol developed for the German miners. In the Czech Republic, it might be possible to establish biobanks; however, a feasibility study is necessary to evaluate potential limiting factors. The feasibility of similar approaches has to be checked in the Canadian cohorts.

Biobanks provide the possibility to develop and investigate markers for chronic exposure, markers for radiation susceptibility and markers of effects. The miner cohorts offer the possibility to investigate radiation susceptibility in relation to lung cancer development. A pathology archive was implemented in Germany many years ago and comparisons of the genetic background of long-term survivors and lung cancer cases according to their radiation exposure can be envisaged using these samples, and genetic predispositions may be detected. In addition, recently developed 'omics tools' may allow the determination of new biological pathways affected by the radiation exposure, which lead to cancer and non-cancer diseases.

\section{Collaboration issues}

In Europe, a tight collaboration between Czech, French, and German investigators already exists, based on several former common EC projects on uranium miners 
(Tirmarche et al., 2003; Tomasek et al., 2008; Tirmarche et al., 2010). Presently, the pooling of data from the three European and three Canadian miner studies is in preparation. The pooled study should include more than 130,000 radon-exposed miners with individual data on exposure. The long follow-up and high statistical power will allow the analyses of cancer and non-cancer effects in relation to low dose radiation. Particular focus should be given to lung cancer, leukaemia, some other non-respiratory cancers and cardiovascular diseases. There is suggestive evidence of an association between indoor radon and leukaemia in children. Miners are exposed to internal and external radiation. Thus, biological effects of different radiation quality could be investigated. In Germany the establishment of a biobank is ongoing. Other countries might follow. A common biobank would enhance the statistical power to perform e.g. studies on genetic-based radiation susceptibility with a suspected small effect size. In addition, new hypotheses about non-cancer disease risk could be tested. A close cooperation of epidemiologists, biologists, dosimetrists and modellers is necessary for such new collaboration activities.

\section{General discussion and perspectives}

As can be seen from the text above, there are several potential study populations that could be used to improve the estimation of the magnitude of cancer risk following internal exposure. It was noted that often internal exposures are to low doses and are of a chronic low dose rate in nature. Thus, these situations are important for issues relating to low dose/dose-rate risk and for radiation protection.

The epidemiological cohorts that were discussed during the workshop cover a large variety of exposed populations and constitute an important resource for the radiation risk research community. The great potential of these studies for quantifying risk from radiation exposure at low doses, even without biological input, must be emphasised.

By its nature, internal contamination risk research is multidisciplinary, requiring input from epidemiology, radiation dosimetry, radiation biology, toxicology, and risk modelling amongst others. The topic of internal contamination risk therefore provides an excellent opportunity to foster and develop further multidisciplinary low dose risk research, and the lively discussions at the workshop confirmed that there were opportunities and enthusiasm for such multidisciplinary research. Epidemiology can be limited by statistical power as a consequence of cohort size and exposure levels. However, it is important to recognise that biological approaches can face similar limitations, especially when considering animal studies. Integration of epidemiological and biological approaches was therefore seen to be potentially of great value. 
It appears that for current studies of internal contamination, the main issues are first linked to exposure and dose reconstruction. Compared with external radiation exposure, many issues are linked to the reconstruction of exposures and doses due to internal contamination (retrospective reconstruction of exposure, biokinetic and dosimetric models, etc.). Also, the variability of exposure situations underlines the need for careful and comprehensive characterisation of the exposures (types of compounds, chemical form, solubility, toxicity, etc.). A combination of different approaches (JEM, bioassay monitoring, retrospective dosimetry, etc.) could be very valuable in order to improve the precision of exposure assessment. Furthermore, internal contamination is concomitant to external exposure (nuclear workers or uranium miners), except for a few exceptions (such as medical uses of 131-Iodine), and generally the contamination does not involve only one radionuclide (iodine and caesium in the Chernobyl exposed populations, caesium and strontium in the Techa River cohort, etc.). These mixed-exposure situations make it more difficult to distinguish the specific effects of each of the exposure components, and complicate the quantification of cancer risk associated with internal contamination. Analyses of such exposure situations require a tight collaboration between epidemiologists, dosimetrists and exposure assessment experts. For instance, the collaboration of dosimetrists and epidemiologists in the framework of the alpha-risk research project made it possible to analyse cancer risk associated with organ doses, taking into account both exposures to radon and radon decay products, gamma radiation and long-lived radionuclides due to uranium ore dust (Tirmarche et al., 2010; Marsh et al., 2011).

The type of sample to be collected and stored in order to implement molecular/biomarker epidemiological studies is critical. Clearly, the samples archived will to some extent limit the range of biomarkers that could be applied in the future. If molecular/biomarker studies are to be included - which samples need to be obtained and stored? Worker studies provide the opportunity for repeated samples for the same individual (at the time of the occupational medical check-up), and therefore the possibility for a longitudinal analysis of the evolution of exposure or effect biomarkers. In some situations the opportunities to collect samples are reducing. For instance, underground uranium miners are in most countries few and getting old in age. Costs of collection and storage can be high; ethical considerations require sustainability of tissue archives to be planned in advance. Experimental studies of internal emitter risk have been carried out in the past but there remain uncertainties related to biokinetics and to biological efficiency that need to be addressed. Human and/or animal samples would benefit from being archived until there is a consensus on the best assays to apply. In this context, careful consideration of the sustainability of archives is important. For cohorts with already existing samples, there is an urgent need to verify the quality 
of available material and to assess its usefulness regarding the identification of biomarkers using recent methods.

In attempting to prioritise the internal contamination research issue(s) of greatest importance and likely benefit for public health and radiation protection, many factors have to be taken into account. Political or public pressure may bear on the prioritisation of research needs. For example, calls for follow-up of those exposed as a consequence of the Fukushima incident were made rapidly. Issues of widespread environmental contamination may be viewed as an urgent need. In these situations researchers should be clear on the likely power of such studies to detect excess risk and the long time required to deliver results. Based on the discussions within the working groups, there was no one clearly over-riding priority area identified. However, the following were seen as priorities:

- Working group on nuclear workers: (i) pooling of French, Belgian, and UK worker uranium studies and testing the relevance and feasibility of biological sampling; (ii) although tritium was not discussed in detail during the workshop, studies of tritium risk, by pooling data from different European nations could also be beneficial to assess the health impact of such exposures, especially in the light of the possible extensive use of tritium in fusion energy production, and (iii) there are clear possibilities for experimental work that could complement an epidemiological approach but biological data from exposed workers are relatively scarce.

- Working group on Chernobyl liquidators and the Techa River: (i) studies of 131-Iodine risk for thyroid cancer among Chernobyl liquidators and cohorts of children in Belarus and Ukraine with direct thyroid activity measurements, and (ii) further studies of leukaemia risk in relation to blood parameters in the Techa River population would be valuable.

- Working group on uranium miners: (i) pooling of German, French and Czech European data (and possibly other data such as that from Canada) to assess the risk of leukaemia following radon exposure; (ii) extensive animal studies have been carried out before, and the availability of collected biological material has to be evaluated, and (iii) the potential to look for leukaemia-associated translocations in blood from ex- or recent miners is of interest but would need careful planning and assessment of likely power to deliver reliable results.

Several of these studies have the potential to be used to investigate other endpoints, such as individual susceptibility or non-cancer effects. For example, there are opportunities to study transgenerational effects and risk of cardiovascular diseases in the Techa River cohort if information on confounders could be obtained.

Any one of these projects would require substantial resources beyond those available within the DoReMi Network. However, further development of the 
research topics above can be expected to foster multidisciplinary working while improving the evidence base on which to develop radiation protection standards and policy relevant to internal contamination.

\section{Conclusion}

In conclusion, this workshop confirmed the importance of research on the health effects of internal contaminations. Epidemiological studies exist, and several with good individual data provide a real possibility to improve the quantification of cancer risk associated with internal emitters. One main limitation is exposure and dose estimator accuracy. Collaboration between dosimetrists, exposure assessment experts, industrial hygienists, statisticians (modelling of measurement errors) and epidemiologists should be able to improve the quantification of the exposure-risk relationship for cancer in the low dose range. Several possible protocols of collaborative research projects between epidemiology and biology were discussed during the workshop. Biological samples do exist for several of these cohorts, but the availability, the quality, and the usefulness of these samples have to be assessed. A few studies have already been launched with these objectives: at the URCRM in Russia on the Techa River cohort and at the BfS in Germany on the Wismut uranium miners. In the near future, further feasibility projects should be proposed and supported in Europe in the framework of the DoReMi network and the MELODI initiative.

Acknowledgements. The workshop was organised in the framework of the DoReMi Network of Excellence. The research leading to these results received funding from the European Atomic Energy Community's Seventh Framework Programme (FP7/2007-2011) under grant agreement $n^{\circ} 249689$. The authors would like to thank the invited speakers for the quality of their presentations, and all workshop participants for their active contribution to the fruitful discussions.

\section{REFERENCES}

AGIR (2010) Circulatory disease risk, Report of the Independent Advisory Group on Ionising Radiation. Health Protection Agency (HPA), Chilton. RCE 16: 1-116.

Akleev A.V., Aleshchenko A.V., Gotlib V., Kudriashova O.V., Semenova L.P., Serebrianyi A.M., et al. (2007) The adaptive response of first generation offsprings irradiated parents, Radiatsionnaia biologiia, radioecologiia / Rossiiskaia akademiia nauk 47 (5), 550-557.

Akleyev A.V., Akushevich I.V., Dimov G.P., Veremeyeva G.A., Varfolomeyeva T.A., Ukraintseva S.V., et al. (2010a) Early hematopoietic effects of chronic radiation exposure in humans, Health Phys 99 (3), 330-336.

Akleyev A.V., Dimov G.P., Varfolomeyeva T.A. (2010b) Late effects in hemopoiesis and bone tissue among people with incorporated osteotropic isotope 90Sr, Health Phys 98 (6), 819-824. 
Akushevich I.V., Veremeyeva G.A., Dimov G.P., Ukraintseva S.V., Arbeev K.G., Akleyev A.V., et al. (2011) Modeling hematopoietic system response caused by chronic exposure to ionizing radiation, Radiat Environ Biophys 50 (2), 299-311.

Atkinson W.D., Law D.V., Bromley K.J., Inskip H.M. (2004) Mortality of employess of the United Kingdom Atomic Energy Authority, 1946-97, Occup Environ Med 61 (7), 577-585.

BEIR-VI (1999) National Research Council, Committee on Health Risks of Exposure to Radon. Health effects of exposure to radon (BEIR VI), National Academy Press, Washington DC.

Bijwaard H., Dekkers F. (2007) Bone cancer risk of (239)pu in humans derived from animal models, Radiat Res 168 (5), 582-592.

Boice J.D., Cohen S.S., Mumma M.T., Dupree Ellis E.A., Eckerman K.F., Leggett R.W., et al. (2011) Mortality among Radiation Workers at Rocketdyne (Atomic International), 1948-2008, Radiat Res 175, 98-115.

Bonatera G.A., Zügel S., Kinscherf R. (2010) Novel systemic cardiovascular disease biomarkers, Current Molecular Medicine 10, 180-205.

Cardis E., Hatch M. (2011) The Chernobyl accident--an epidemiological perspective, Clin Oncol (Royal College of Radiologists (Great Britain)) 23 (4), 251-260.

Cardis E., Pernot E. (2011) Overview of the existing epidemiological cohorts of European populations exposed to occupational, environmental or medical low dose radiations, DoReMi. Deliverable 4.1.

Carpenter L.M., Higgins C.D., Douglas A.J., Maconochie N.E., Omar R.Z., Fraser P., et al. (1998) Cancer mortality in relation to monitoring for radionuclide exposure in three UK nuclear industry workforces, Br J Cancer 78 (9), 1224-1232.

Chan C., Hughes T.S., Muldoon S., Aldrich T., Rice C., Hornung R., et al. (2010) Mortality patterns among Paducah Gaseous Diffusion Plant workers, J Occup Environ Med 52 (7), 725-732.

Darby S., Hill D., Deo H., Auvinen A., Barros-Dios J.M., Baysson H., et al. (2006) Residential radon and lung cancer--detailed results of a collaborative analysis of individual data on 7148 persons with lung cancer and 14,208 persons without lung cancer from 13 epidemiologic studies in Europe, Scand J Work Environ Health 32 (Suppl 1), 1-83.

Drozdovitch V., Khrouch V., Maceika E., Zvonova I., Vlasov O., Bratilova A., et al. (2010) Reconstruction of radiation doses in a case-control study of thyroid cancer following the Chernobyl accident, Health Phys 99 (1), 1-16.

Erdmann J., Linsel-Nitschke, Schunkert H. (2010) Genetic Causes of myocardial infarcation. New insights from genome-wide association studies, Dtsch. Ärztebl Int 107, 694-699.

Garsi J.P., Schlumberger M., Rubino C., Ricard M., Labbe M., Ceccarelli C., et al. (2008) Therapeutic administration of $131 \mathrm{I}$ for differentiated thyroid cancer: radiation dose to ovaries and outcome of pregnancies, J Nucl Med 49 (5), 845-852.

Guseva Canu I., Ellis E.D., Tirmarche M. (2008a) Cancer risk in nuclear workers occupationally exposed to uranium-emphasis on internal exposure, Health Phys 94 (1), 1-17.

Guseva Canu I., Molina G., Goldberg M., Collomb P., David J.C., Perez P., et al. (2008b) Development of a job exposure matrix for the epidemiological follow-up of workers in the French nuclear industry, Rev Epidémiol Santé Publique 56 (1), 21-29.

Guseva Canu I., Paquet F., Goldberg M., Auriol B., Berard P., Collomb P., et al. (2009) Comparative assessing for radiological, chemical, and physical exposures at the French uranium conversion plant: Is uranium the only stressor?, Int J Hyg Environ Health 212, 398-413.

Guseva Canu I., Jacob S., Cardis E., Wild P., Caer-Lorho S., Auriol B., et al. (2010a) Reprocessed uranium exposure and lung cancer risk, Health Phys 99 (3), 308-313.

Guseva Canu I., Laurier D., Caër-Lorho S., Samson E., Tirmarche M., Auriol B., et al. (2010b) Characterisation of protracted low-level exposure to uranium in the workplace: A comparison of two approaches, Int J Hyg Environ Health 213, 270-277. 
Guseva Canu I., Jacob S., Caer-Lorho S., Cardis E., Wild P., Garsi J.P., et al. (2011) Uranium carcinogenicity in humans might depend on the physical and chemical nature of uranium and its isotopic composition: Results from pilot epidemiological study of French nuclear workers, Cance Cause Control (in press).

Harrison J. (2009) Biokinetic and dosimetric modelling in the estimation of radiation risks from internal emitters, J Radiol Prot. 29 (2A), A81-A105.

HLEG (2009) High Level and Expert Group (HLEG). HLEG Report on European Low Dose Risk Research. EUR 23884. Luxembourg, UK: Office for Official Publications of the European Communities; 2009.

Hofer E. (2008) How to account for uncertainty due to measurement errors in an uncertainty analysis using Monte Carlo simulation, Health Phys 95 (3), 277-290.

Hofmann W., Crawford-Brown D.J., Fakir H. and Monchaux G. (2006) Modeling lung cancer incidence in rats following exposure to radon progeny, Radiat Prot Dosim 122 (1-4), 345-348.

Kesminiene A., Evrard A.-S., Ivanov V.K., Malakhova I.V., Kurtinaitis J., Stengrevics A., et al. Risk of thyroid cancer among Chernobyl liquidators., Radiat Res. (Submitted).

Kossenko M.M., Thomas T.L., Akleyev A.V., Krestinina L.Y., Startsev N.V., Vyushkova O.V., et al. (2005) The Techa River Cohort: study design and follow-up methods, Radiat Res 164 (5), 591601.

Kreuzer M., Schnelzer M., Tschense A., Walsh L., Grosche B. (2010) Cohort profile: the German uranium miners cohort study (WISMUT cohort), 1946-2003, Int J Epidemiol 39 (4), 980-987.

Kryuchkov V., Chumak V., Maceika E., Anspaugh L.R., Cardis E., Bakhanova E., et al. (2009) Radrue method for reconstruction of external photon doses for Chernobyl liquidators in epidemiological studies, Health Phys 97 (4), 275-298.

Leuraud K., Billon S., Bergot D., Tirmarche M., Caer S., Quesne B., et al. (2007) Lung cancer risk associated to exposure to radon and smoking in a case-control study of French uranium miners, Health Phys 92 (4), 371-378.

Leuraud K., Schnelzer M., Tomasek L., Hunter N., Timarche M., Grosche B., et al. (2011) Radon, Smoking and Lung Cancer Risk: Results of a Joint Analysis of Three European Case-Control Studies Among Uranium Miners, Radiat Res 176, 375-387.

Little M.P., Tawn E.J., Tzoulaki I., Wakeford R., Hildebrandt G., Paris F., et al. (2010) Review and meta-analysis of epidemiological associations between low/moderate doses of ionizing radiation and circulatory disease risks, and their possible mechanisms, Radiat Environ Biophys, 49 (2), 139-153.

Marsh J.W., Bessa Y., Birchall A., Blanchardon E., Hofmann W., Nosske D., et al. (2008) Dosimetric models used in the Alpha-Risk project to quantify exposure of uranium miners to radon gas and its progeny, Radiat Prot Dosim 130 (1), 101-106.

Marsh J.W., Blanchardon E., Gregoratto D., Hofmann W., Karcher K., Nosske D., et al. (2011) Dosimetric Calculations for Uranium Miners for Epidemiological Studies, Radiat Prot Dosim (Epub ahead of print).

McGeoghegan D., Binks K., Gillies M., Jones S., Whaley S. (2008) The non-cancer mortality experience of male workers at British Nuclear Fuels plc, 1946-2005, Int J Epidemiol 37 (3), 506518.

Monchaux G., Morlier J.P., Altmeyer S., Debroche M., Morin M. (1999) Influence of exposure rate on lung cancer induction in rats exposed to radon progeny, Radiat Res 152 (6 Suppl), S137-140.

Monchaux G., Morlier J.P., Morin M., Chameaud J., Lafuma J., Masse R. (1994) Carcinogenic and cocarcinogenic effects of radon and radon daughters in rats, Environ Health Perspect 102 (1), 64-73.

Ory C., Ugolin N., Levalois C., Lacroix L., Caillou B., Bidart J.M., et al. (2011) Gene expression signature discriminates sporadic from post-radiotherapy-induced thyroid tumors, Endocr Relat Cancer 18 (1), 193-206. 
Ostroumova E., Brenner A., Oliynyk V., McConnell R., Robbins J., Terekhova G., et al. (2009) Subclinical hypothyroidism after radioiodine exposure: Ukrainian-American cohort study of thyroid cancer and other thyroid diseases after the Chornobyl accident (1998-2000), Environ Health Perspect 117 (5), 745-750.

Paquet F., Barbey P., Bardiès M., Biau A., Blanchardon E., Chetioui A., et al. (2011) L'évaluation et la gestion des risques associés aux expositions aux radionucléides émetteurs Auger et bêta. Avis et propositions de pistes de Recherche. Rapport IRSN/ DS 2010-03. Institut de Radioprotection et de Sûreté Nucléaire. Fontenay-aux-Roses.

Port M., Boltze C., Wang Y., Roper B., Meineke V., Abend M. (2007) A radiation-induced gene signature distinguishes post-Chernobyl from sporadic papillary thyroid cancers, Radiat Res 168 (6), 639-649.

Priest N.D., Hoel D. (2010) Determining the relative toxicity and RBE of internal emitters in animals, Health Phys 99 (3), 367-370.

Puncher M., Birchall A. (2008) A Monte Carlo method for calculating Bayesian uncertainties in internal dosimetry, Radiat Prot Dosim 132 (1), 1-12.

Rubino C., de Vathaire F., Dottorini M.E., Hall P., Schvartz C., Couette J.E., et al. (2003) Second primary malignancies in thyroid cancer patients, Br J Cancer 89 (9), 1638-1644.

Schnelzer M., Hammer G.P., Kreuzer M., Tschense A., Grosche B. (2010) Accounting for smoking in the radon-related lung cancer risk among German uranium miners: results of a nested casecontrol study, Health Phys 98 (1), 20-28.

Sokolnikov M.E., Gilbert E.S., Preston D.L., Ron E., Shilnikova N.S., Khokhryakov V.V., et al. (2008) Lung, liver and bone cancer mortality in Mayak workers, Int J Cancer 123 (4), 905-911.

SOLO-project. SOLO: Epidemiological Studies of Exposed Southern Urals Populations. Integrated Project FP7-249675 (http://solo-fp7.eu).

SOUL-project. SOUL: Southern Urals Radiation Risk Research. Integrated Project FP6-516478 (http://www.helmholtz-muenchen.de/soul/index_new.htm).

Thierry-Chef I., Bérard P., Bingham D., Blanchardon E., Birchall A., Bull R., et al. (2010) Approach to derive doses for case-control studies of lung cancer and leukaemia among workers internally exposed to uranium and plutonium, IRPA-12.

Tirmarche M., Laurier D., Bergot D., Billon S., Brueske-Hohlfeld I., Collier C., et al. (2003) Quantification of lung cancer risk after low radon exposure and low exposure rate: synthesis from epidemiological and experimental data. Final scientific report, February 2000- July 2003. Contract FIGH-CT1999-00013. Brussels, European Commission DG XII. Euratom Research Projects and Training Activities (Volume I).

Tirmarche M., Laurier D., Bochicchio F., Cardis E., Binks K., Hofmann W., et al. (2010) Final scientific report of the Alpha Risk Project (web site: http://www.alpha-risk.org) funded by the European Commission EC FP6 (ref. FI6R-CT-2005-516483). Brussels, European Commission DG XII.

Tolstykh E.I., Degteva M.O., Peremyslova L.M., Shagina N.B., Shishkina E.A., Krivoshchapov V.A., et al. (2011) Reconstruction of long-lived radionuclide intakes for Techa riverside residents: strontium-90, Health Phys 101 (1), 28-47.

Tomasek L. (2011) Interaction of radon and smoking among Czech uranium miners, Radiat Prot Dosim 145 (2-3), 238-242.

Tomasek L., Rogel A., Tirmarche M., Mitton N., Laurier D. (2008) Lung cancer in French and Czech uranium miners: Radon-associated risk at low exposure rates and modifying effects of time since exposure and age at exposure, Radiat Res 169 (2), 125-137.

Tronko M.D., Howe G.R., Bogdanova T.I., Bouville A.C., Epstein O.V., Brill A.B., et al. (2006) A cohort study of thyroid cancer and other thyroid diseases after the Chernobyl accident: thyroid cancer in Ukraine detected during first screening, J Nat Cancer Instit 98 (13), 897-903. 
Tuttle R.M., Vaisman F. and Tronko M.D. (2011) Clinical presentation and clinical outcomes in Chernobyl-related paediatric thyroid cancers: what do we know now? What can we expect in the future?, Clin Oncol (Royal College of Radiologists (Great Britain)) 23 (4), 268-275.

UNSCEAR (2008) United Nations Scientific Committee on the Effects of Atomic Radiation (UNSCEAR). UNSCEAR 2006 Report to the General Assembly with Scientific Annexes, Effects of Ionizing Radiation. Vol. 1: Report and Annexes A and B New York, NY: United Nations.

UNSCEAR (2011) United Nations Scientific Committee on the Effects of Atomic Radiation. UNSCEAR 2008 Report, Annex D - Health effects due to radiation from the Chernobyl accident, United Nations, New York.

Vacquier B., Caer S., Rogel A., Feurprier M., Tirmarche M., Luccioni C., et al. (2008) Mortality risk in the French cohort of uranium miners: extended follow-up 1946-1999, Occup Environ Med 65 (9), 597-604.

van Dillen T., Dekkers F., Bijwaard H., Kreuzer M., Grosche B. (2011) Lung cancer from radon: a twostage model analysis of the WISMUT Cohort, 1955-1998, Radiat Res 175 (1), 119-130.

Weber D.G., Casjens S., Rozynek P., Lehnert M., Zilch-Schoneweis S., Bryk O., et al. (2010) Assessment of mRNA and microRNA Stabilization in Peripheral Human Blood for Multicenter Studies and Biobanks, Biomark Insights 5, 95-102.

Yiin J.H., Anderson J.L., Daniels R.D., Seel E.A., Fleming D.A., Waters K.M., et al. (2009) A nested case-control study of multiple myeloma risk and uranium exposure among workers at the Oak Ridge Gaseous Diffusion Plant, Radiat Res 171 (6), 637-645.

Zablotska L.B., Ron E., Rozhko A.V., Hatch M., Polyanskaya O.N., Brenner A.V., et al. (2010) Thyroid cancer risk in Belarus among children and adolescents exposed to radioiodine after the Chornobyl accident, Br J Cancer 104 (1), 181-187.

Zablotska L.B., Ron E., Rozhko A.V., Hatch M., Polyanskaya O.N., Brenner A.V., et al. (2011) Thyroid cancer risk in Belarus among children and adolescents exposed to radioiodine after the Chornobyl accident, Br J Cancer 104 (1), 181-187. 